\title{
Computing generalized Nash equilibria by polynomial programming
}

\author{
Couzoudis, Eleftherios ; Renner, Philipp
}

\begin{abstract}
We present a new way to solve generalized Nash equilibrium problems. We assume the feasible set to be compact. Furthermore all functions are assumed to be polynomials. However we do not impose convexity on either the utility functions or the action sets. The key idea is to use Putinar's Positivstellensatz, a representation result for positive polynomials, to replace each agent's problem by a convex optimization problem. The Nash equilibria are then feasible solutions to a system of polynomial equations and inequalities. Our application is a model of the New Zealand electricity spot market with transmission losses based on a real dataset
\end{abstract}

DOI: https://doi.org/10.1007/s00186-012-0422-5

Posted at the Zurich Open Repository and Archive, University of Zurich ZORA URL: https://doi.org/10.5167/uzh-156520

Journal Article

Published Version

Originally published at:

Couzoudis, Eleftherios; Renner, Philipp (2013). Computing generalized Nash equilibria by polynomial programming. Mathematical Methods of Operations Research, 77(3):459-472.

DOI: https://doi.org/10.1007/s00186-012-0422-5 


\title{
Computing generalized Nash equilibria by polynomial programming
}

\author{
Eleftherios Couzoudis · Philipp Renner
}

Received: 23 November 2012 / Accepted: 27 November 2012 / Published online: 3 January 2013

(C) Springer-Verlag Berlin Heidelberg 2013

\begin{abstract}
We present a new way to solve generalized Nash equilibrium problems. We assume the feasible set to be compact. Furthermore all functions are assumed to be polynomials. However we do not impose convexity on either the utility functions or the action sets. The key idea is to use Putinar's Positivstellensatz, a representation result for positive polynomials, to replace each agent's problem by a convex optimization problem. The Nash equilibria are then feasible solutions to a system of polynomial equations and inequalities. Our application is a model of the New Zealand electricity spot market with transmission losses based on a real dataset.
\end{abstract}

Keywords Generalized nash equilibrium - Parametrized optimization · Real algebraic geometry · Nonconvex optimization · Electricity spot market · Transmission loss

\section{Introduction}

There has been a lot of interest in the computation of normalized Nash equilibria since Rosen (1965) introduced them. In essence the approach is to reformulate the problem either as a variational inequality or using penalty-functions or Nikaido-Isoda-typefunctions. The one thing all computational papers have in common is the assumption

We thank Didier Aussel, Hans-Jakob Lüthi, Cordian Riener and two anonymous referees.

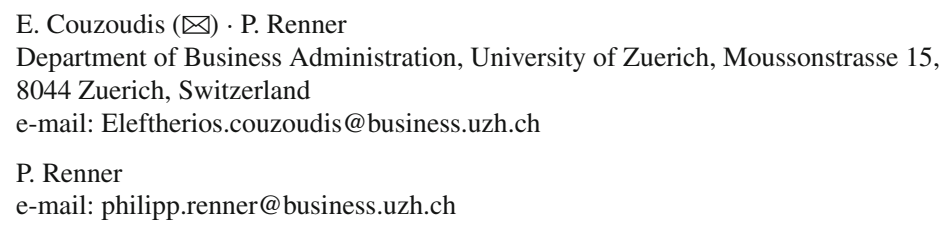


of player-convexity on the utility functions of the players (Facchinei and Kanzow 2007).

There are some attempts in the literature to extend the solution approach to quasi-convex problems. However error bounds are only provided under some strong monotonicity assumptions (Aussel et al. 2011a; Aussel and Dutta 2011).

Unlike the usual approaches to generalized Nash equilibrium problems (GNEPs), we do not need any convexity assumptions on our functions and sets. We are also not restricted to normalized equilibria. However, since we employ tools from real algebraic geometry we require every constraint and objective function to be polynomial. Note that the KKT conditions do not provide sufficient conditions in the case of non-convex function. Thus, instead of the usual approach, we replace each agent's problem with the convex relaxation obtained by Putinar's Positivstellensatz. Each of these problems is then a parametrized semi-definite optimization problem. The corresponding optimality conditions however are a system of polynomial equations and inequalities. We show that some equilibria are feasible points to this system and that any other feasible point is almost optimal. In case where we have a representation for a non-negative polynomial, only equilibria are feasible points. We find those points with the solver Ipopt.

We also could do this with just slight modification for rational functions. The relevant theorems to apply this approach for rational functions can be found in Jibetean and de Klerk (2006). Our attention is on a non-cooperative, single stage game in normal form, a onetime situation without reoccurrence and a finite number of players who move simultaneously.

Let $N \in \mathbb{N}$ be the finite number of players in the examined $N$-person game. Every player $v \in I$ with $I:=\{1, \ldots, N\}$ chooses his strategy $x^{v}$ from the strategy set $X_{v}\left(x^{-v}\right) \subseteq \mathbb{R}^{n_{v}}$, where $n_{v}$ is a positive integer. For the sake of simplicity the strategy set of all other players except $v$ is given by $x^{-v}:=\left(x^{\nu^{\prime}}\right)_{v^{\prime}=1, v^{\prime} \neq v}^{N} \in \mathbb{R}^{n_{-v}}$. The complete strategy vector of all players is specified with $x:=\left(x^{\nu}\right)_{\nu=1}^{N} \in \mathbb{R}^{n}$ and $n:=\sum_{v=1}^{N} n_{v}$. Hence $n_{-v}=n-n_{v}$ and therefore the tuple of strategies for the whole game has dimension $n$ :

$$
x:=\left(x^{v}, x^{-v}\right)^{T}=\left(x^{1}, \ldots, x^{v-1}, x^{v}, x^{\nu+1}, \ldots, x^{N}\right)^{T} \in \mathbb{R}^{n}
$$

The scope of action $X_{v}\left(x^{-v}\right)$ for every player $v$ in this game is influenced by the strategies of the opponents $x^{-v}$. For $v=1, \ldots, N$ let $X_{v}: \mathbb{R}^{n_{-v}} \rightrightarrows \mathbb{R}^{n_{v}}$ be a pointto-set-mapping and for every fixed $x^{-v}$ a subset of $\mathbb{R}^{n_{v}}$. The allowed strategy set of the player $v$ has then the following form:

$$
X_{v}\left(x^{-v}\right):=\left\{x^{v} \mid\left(x^{v}, x^{-v}\right) \in X\right\}
$$

Thereby $X \subseteq \mathbb{R}^{n}$ is assumed to be nonempty and compact which implies the compactness of every set $X_{v}\left(x^{-v}\right)$. For this work $X$ has the following structure

$$
X:=\left\{x \in \mathbb{R}^{n} \mid g_{v}(x) \geq 0, h_{v}\left(x^{\nu}\right) \geq 0 \forall v=1, \ldots, N\right\}
$$


where the functions $g_{v}: \mathbb{R}^{n} \rightarrow \mathbb{R}^{l_{v}}$ are constraints influenced by other players, $h_{v}: \mathbb{R}^{n_{v}} \rightarrow \mathbb{R}^{m_{v}}$ are constraints specific to each player $v$ and $l_{v}, m_{v}$ are positive integers. Combining the two set declarations $X$ and $X_{v}$ gives us

$$
X_{v}\left(x^{-v}\right):=\left\{x^{v} \mid g_{v}\left(x^{v}, x^{-v}\right) \geq 0, h_{v}\left(x^{v}\right) \geq 0\right\} \quad \forall v=1, \ldots, N
$$

and at the same time the possibility to define the feasible set for any point $x \in \mathbb{R}^{n}$ :

$$
\Omega(x):=X_{1}\left(x^{-1}\right) \times \cdots \times X_{N}\left(x^{-N}\right) .
$$

The last and yet missing basic element is the payoff. The assessment of the player's strategy set $X_{v}$ and therefore the choice of action $x^{v}$ of player $v$ depends on the corresponding utility or payoff function $\theta_{v}: \mathbb{R}^{n} \rightarrow \mathbb{R}$. A finite $N$-person game is defined by the triple $\left(I,\left(X_{v}\right)_{v \in I},\left(\theta_{v}\right)_{v \in I}\right)$.

Furthermore a theoretical construct is needed to decide which player choices are rational and optimal. In this case the payoff function $\theta_{v}$ is assumed to be a cost or loss function. Every player $v$ tries to minimize his loss given the exogenous decision of the competition:

$$
\begin{array}{r}
R_{v}\left(x^{-v}\right): \min _{x^{v} \in \mathbb{R}^{n_{v}}} \theta_{v}\left(x^{v}, x^{-v}\right) \\
\text { s.t. } \quad x^{v} \in X_{v}\left(x^{-v}\right) .
\end{array}
$$

The solution set mapping $R_{v}$ is also the best-response mapping of player $\nu$.

Definition 1 A strategy $x^{\star} \in \Omega\left(x^{\star}\right)$ is a generalized Nash equilibrium (GNE), if and only if $x^{\star, v}$ satisfies the following inequality:

$$
\theta_{v}\left(x^{\star, v}, x^{\star,-v}\right) \leq \theta_{v}\left(x^{v}, x^{\star,-v}\right) \quad \forall x^{v} \in X_{v}\left(x^{\star,-v}\right), \forall v \in\{1, \ldots, N\} .
$$

The Nash Equilibrium is therefore, for any player $v$, the optimal decision given the expected choice $x^{\star,-v}$ of the fellow players.

\section{The model}

"The [Electricity] Authority [of New Zealand] is responsible for ensuring the effective day-to-day operation of the electricity system and markets through the operation of core system and market services in accordance with the [Electricity Industry Participation] Code [2010]." ${ }^{1}$ The corresponding tasks are assigned to these market operation service providers: Registry Manager, Reconciliation Manager, Pricing Manager, Clearing Manager, Information System Manager, System Operator and Inter-island Financial Transmission Rights Manager. ${ }^{2}$ Our focus here lies on the Independent System Operator (ISO) and on Part 13 of the

\footnotetext{
1 Electricity Authority NZ (2012).

2 Electricity Authority NZ (2012).
} 
Electricity Industry Participation Code 2010 which sets out the trading arrangements.

New Zealand consists of two main islands, the energy demanding north and the energy producing south with a high voltage direct current (HVDC) Link between them. Our highly abstract network is therefore composed of only two nodes and two directed edges. The arc $t_{1}$ is directed from the north island to the south island and $t_{2}$ is the inverted arc of $t_{1}$. The power flow capacity of both arcs is $\bar{t}$.

"Each [Energy Producer (EP) or] generator ... must submit an offer to the system operator for each trading period in the schedule period, under which the generator is prepared to sell electricity to the clearing manager, and ensure that the system operator receives an offer at least 71 trading periods before the beginning of the trading period to which the offer applies."3 An offer submitted by a generator may have a maximum of 5 price bands for each trading period and may not exceed, for each trading period, the generators reasonable estimate of the quantity of electricity capable of being supplied at that node. The price offered in each band must increase progressively from band to band as the aggregate quantity increases. An exception are intermittent generators with a maximum of 1 price band for each trading period and co-generators with a maximum of 2 price bands for each trading period. ${ }^{4}$ "For each price band, an ... offer must specify a quantity expressed in megawatt [MW] to not more than 3 decimal places. The minimum quantity that may be bid or offered in a price band for a trading period is $0.000 \mathrm{MW}$." "Prices in ... offers must be expressed in dollars and whole cents per megawatthour $[\mathrm{MWh}] \ldots$ There is no upper limit on the prices that may be specified and the lower limit is $\$ 0.00$ per MWh ...."6

It is clear that the inverse supply function of the power producers would be piecewise linear and an extension to the here presented model is straightforward. In the literature the approach for the New Zealand electricity spot market is to make an quadratic approximation of the inverse supply function. This is shown in Aussel et al. (2011b) which in turn is based on Hobbs et al. (2000) and yields in Aussel's example depending on the control variables quadratic or even non-convex cubic polynomials as revenue functions.

For this proof of concept we restrict the offers from energy producers to one price band with a lower level of zero and a static demand $\delta_{N}$ for the north island and $\delta_{S}$ for the south island. Hence, in this scenario there are only two kinds of players, the Independent System Operator and the Energy Producers. The price per Gigawatt-hour [GWh] of each EP is denoted by $a^{v}$ and $q^{v}$ is the maximum amount of power offered in GWh. The ISO can choose for any energy offer $q^{\nu}$ the effective fraction $c^{\nu}$. Then the revenue function for each EP is given by $a^{v} c^{v} q^{v}$. All terms are with respect to one

\footnotetext{
3 (Electricity Authority NZ 2010b, 13.6).

4 (Electricity Authority NZ 2010b, 13.9, 13.12).

5 (Electricity Authority NZ 2010b, 13.16).

6 (Electricity Authority NZ 2010b, 13.15).
} 
time period which is one day. This is in accordance to the pricing manager who sets the final prices on a daily basis.

The ISO wants to achieve the social optimum in minimizing the expenditure for the needed energy and the loss through transporting energy.

$$
\begin{aligned}
\min _{c^{\nu}, t_{1}, t_{2}} & \sum_{\nu=1}^{N} a^{v} c^{v} q^{\nu} \\
\text { s.t. } & \sum_{\nu=1}^{i} c^{v} q^{\nu}-t_{1}-\lambda\left(t_{1}\right)^{2}+t_{2}-\lambda\left(t_{2}\right)^{2}-\delta_{N} \geq 0 \\
& \sum_{\nu=i+1}^{N} c^{v} q^{\nu}-t_{2}-\lambda\left(t_{2}\right)^{2}+t_{1}-\lambda\left(t_{1}\right)^{2}-\delta_{S} \geq 0 \\
& 0 \leq c^{\nu} \leq 1 \quad \forall v=1, \ldots, N \\
& \bar{t} \geq t_{1} \geq 0, \quad \bar{t} \geq t_{2} \geq 0 .
\end{aligned}
$$

The objective function is linear and the constraint set is convex, so the ISO problem is clearly convex. Both network constraints are pretty self explanatory except for the quadratic terms $\lambda\left(t_{\{1,2\}}\right)^{2}$. Transmitting power is not lossless hence we form a term for the arising heat loss using Joule's and Ohm's laws (Tipler et al. 2000):

$$
\left.\begin{array}{l}
P=U I \\
P_{\text {loss }}=I^{2} R
\end{array}\right\} P_{\text {loss }}=R \cdot\left(\frac{P}{U}\right)^{2}=R^{\prime} \cdot l \cdot\left(\frac{P}{U}\right)^{2}=\frac{R^{\prime} \cdot l}{U^{2}} \cdot P^{2}
$$

The unit of $P$ is watt and not equal to watt hour unit of $t_{\{1,2\}}$ thus a conversion is necessary:

$$
\frac{R^{\prime} \cdot l}{U^{2}} \cdot\left(\frac{P_{h}}{24}\right)^{2} \cdot 24=\frac{R^{\prime} \cdot l}{U^{2} \cdot 24} \cdot\left(P_{h}\right)^{2}=\lambda \cdot\left(P_{h}\right)^{2}
$$

The meanings of the different terms are:

$$
\begin{array}{ll}
P: \text { power } & R \text { : electrical resistivity at } 20^{\circ} \mathrm{C} \\
U: \text { voltage } & R^{\prime}: \text { electrical resistivity } / \mathrm{km} \text { at } 20^{\circ} \mathrm{C} \\
I: \text { current } & l: \text { length of transmission line. }
\end{array}
$$

For the cost function of the EPs we seek a technology-driven approach. If something is burned up, the produced heat can be measured in Joule $[\mathrm{J}]$. The heat conversion rate $\eta_{v}$ now answers the question how much of that heat can be converted into electric energy. This principle can of course be extended into non heat based technologies. The player-specific cost function for the EPs has now the following form: 


$$
C_{v}\left(q^{v}\right)=\overbrace{\left(\eta_{v} \cdot q^{v}\right)}^{G J / G W h \times G W h} \cdot \underbrace{\left(\phi_{v}+\tau_{v}\right)}_{\text {fuel price: } \$ / G J}+\overbrace{o_{v}}^{\$ / G W h} q^{v} .
$$

The parameter $\eta_{v}$ is technology dependent. The fuel price consists of the commodity price $\phi_{\nu}$ and the delivery costs $\tau_{\nu}$. The coefficient for other variable costs such as operation and maintenance is denoted as $o_{v}$. Furthermore each EP $v$ has a limited power generating capacity $\kappa_{v}$ given in $G W h$. The optimization problem for the profit maximizing EPs is then as follows:

$$
\begin{aligned}
\max _{a^{v}, q^{v}} & a^{v} c^{v} q^{v}-\left(\eta_{\nu} c^{v} q^{v}\right)\left(\phi_{v}+\tau_{v}\right)-o_{v} c^{v} q^{v} \\
\text { s.t. } & \kappa_{v} \geq q^{v} \geq 0, \\
& a^{v}-\eta_{v}\left(\phi_{v}+\tau_{v}\right)-o_{v} \geq 0, \\
& \sum_{i \neq v}^{N} a^{i} c^{i} q^{i}-a^{v} c^{v} \sum_{i=1}^{N} c^{i} q^{i} \geq 0, \\
& \sum_{i=1}^{N} q^{i}-\lambda\left(\left(t_{1}\right)^{2}+\left(t_{2}\right)^{2}\right)-\delta_{N}-\delta_{S} \geq 0 .
\end{aligned}
$$

A static upper bound on the price is an invitation to a price agreement. All producers would settle at this upper bound. On the other hand competitive behavior is to underbid your opponents, as long as your price is above your marginal costs, and given you are chosen by the ISO. So the two constraints on $a^{v}$ do exactly that. The last constraint is introduced to avoid the infeasibility of the ISO's problem.

Obviously the constraint set is compact. The Eigenvalues of the Hessian of the objective function are $\left\{-c^{v}, c^{v}\right\}$ with $c^{v} \in[0,1]$. Thus the Hessian is indefinite for non trivial $c^{v}$ and therefore this simple model does not have a distinct curvature. With this game we can obviously take full advantage of our approach.

\section{Sum of squares optimization}

In outlining the theory behind our solution approach we follow Laurent (2009) and Lasserre (2010b). We will use the following notations:

$-\mathbb{R}\left[x_{1}, \ldots, x_{n}\right]$ the ring of polynomials in $n$ variables over the real numbers.

$-\boldsymbol{x}=\left(x_{1}, \ldots, x_{n}\right)$ as shorthand notation.

- $f \in \mathbb{R}[\boldsymbol{x}], f(\boldsymbol{x})=\sum_{\alpha} a_{\alpha} \boldsymbol{x}^{\alpha}$ where $\alpha \in \mathbb{N}^{n}$ and $\boldsymbol{x}^{\alpha}=x_{1}^{\alpha_{1}} \cdots x_{n}^{\alpha_{n}}$. Then $\operatorname{deg}(f)=$ $\max _{\left\{\alpha \mid a_{\alpha} \neq 0\right\}}|\alpha|=\max _{\left\{\alpha \mid a_{\alpha} \neq 0\right\}} \sum_{i} \alpha_{i}$.

- For any polynomial $g \in \mathbb{R}[\boldsymbol{x}]$ denote $d_{g}=\left\lceil\frac{\operatorname{deg}(g)}{2}\right\rceil$. 
3.1 Basic definitions and theorems

First we will introduce the class of convex optimization problems that is of interest to us here. To do this we need a few basic notions from linear algebra.

Definition 2 A symmetric matrix $Q \in \mathbb{R}^{n \times n}$ is called positive semidefinite, if and only if $w^{T} Q w \geq 0$ for all $w \in \mathbb{R}^{n}$. It is denoted $Q \succcurlyeq 0$.

In the next proposition we recall a condition for a matrix to be positive semidefinite.

Proposition 1 Let $Q \in \mathbb{R}^{n \times n}$ be a symmetric matrix with rank $m$. Then the following statements are equivalent

(a) $Q$ is positive semidefinite.

(b) There exists a lower triangular matrix $L \in \mathbb{R}^{n \times n}$ with nonnegative diagonal such that $Q=L L^{T}$.

Note here that the equivalent statement for positive semidefiniteness can be expressed by polynomial equations and inequalities.

Now we introduce some basic notions from real algebraic geometry.

Definition 3 Let $g_{1}, \ldots, g_{k} \in \mathbb{R}[\boldsymbol{x}]$. We call the set

$$
K=\left\{\boldsymbol{x} \mid g_{1}(\boldsymbol{x}) \geq 0, \ldots, g_{k}(\boldsymbol{x}) \geq 0\right\}
$$

a basic semi-algebraic set.

Definition 4 (a) A polynomial $\sigma \in \mathbb{R}[\boldsymbol{x}]$ of degree $2 d$ is called a sum of squares, if and only if there exists polynomials $p_{1}, \ldots, p_{m} \in \mathbb{R}[\boldsymbol{x}]$ such that $\sigma=\sum_{i} p_{i}^{2}$.

(b) Let $\Sigma[x] \subset \mathbb{R}[\boldsymbol{x}]$ denote the set of sum of squares.

(c) Let $\Sigma_{2 d}[x] \subset \mathbb{R}[\boldsymbol{x}]$ denote the set of sum of squares up to degree $2 d$.

Since a sum of squares is always a nonnegative function it is easy to see that a polynomial can only be a sum of squares if it has even degree. The question whether a positive polynomial is a sum of squares however is much more involved. There is the following representation result.

Theorem 1 (Putinar's Positivstellensatz) (Lasserre 2010b, Th.2.14) Let $f, g_{1} \ldots, g_{m}$ $\in \mathbb{R}[\boldsymbol{x}]$ be polynomials and

$$
K=\left\{\boldsymbol{x} \in \mathbb{R}^{n} \mid g_{1}(\boldsymbol{x}) \geq 0, \ldots, g_{m}(\boldsymbol{x}) \geq 0\right\} \subset \mathbb{R}^{n}
$$

a semi-algebraic set such that one of the following holds,

- $g_{i}$ are affine and $K$ is a bounded polyhedron.

- For some $j$ the set $\left\{\boldsymbol{x} \in \mathbb{R}^{n} \mid g_{j}(\boldsymbol{x}) \geq 0\right\}$ is compact.

If $f$ is strictly positive on $K$ then

$$
f=\sigma_{0}+\sum_{i=1}^{m} \sigma_{i} g_{i}
$$


for some $\sigma_{0}, \ldots, \sigma_{m} \in \Sigma[x]$.

The conditions for this theorem to hold are not as restrictive as it might seem at first glance. If we know an $N$ such that $\|\boldsymbol{x}\|_{2} \leq N$ for all $\boldsymbol{x} \in K$, we then can add the redundant ball constraint $\sum_{i} x_{i}^{2} \leq N^{2}$. The problem with the theorem is that we do not know the degrees of the coefficients $\sigma_{i}$. There exist bounds on their degree (Lasserre 2010b, Th.2.16), however for practical purposes these are of no use to us.

In an example below we will later see that we can use this kind of representation even without the compactness assumption. Additionally in many models one has an intuitive idea about the form of the solution and can pick an $N$ accordingly.

\subsection{Optimization of polynomials over semialgebraic sets}

Let $p, g_{1}, \ldots, g_{m} \in \mathbb{R}[\boldsymbol{x}]$ and $K=\left\{\boldsymbol{x} \mid g_{1}(\boldsymbol{x}) \geq 0, \ldots, g_{m}(\boldsymbol{x}) \geq 0\right\}$. We want to solve the following optimization problem

$$
\inf _{\boldsymbol{x} \in K} p(\boldsymbol{x}) .
$$

Assuming the supremum over the empty set is $-\infty$, this is equivalent to the following semi infinite optimization problem

$$
\begin{aligned}
& \sup _{\rho} \rho \\
& \text { s.t. } p(\boldsymbol{x})-\rho>0 \forall \boldsymbol{x} \in K
\end{aligned}
$$

In general this is a difficult problem. However there are several representation results for positive polynomials over basic semi-algebraic sets. If $K$ satisfies the conditions of Theorem 1 then we can reformulate the problem into

$$
\begin{aligned}
& \sup _{\rho, \sigma_{0}, \sigma_{1}, \ldots, \sigma_{m}} \rho \\
& \text { s.t. } p-\rho=\sigma_{0}+\sum_{i} \sigma_{i} g_{i} \\
& \sigma_{0}, \sigma_{i} \in \Sigma[x] .
\end{aligned}
$$

Note that the equality here means equality as polynomial functions. This is equivalent to the coefficients on the left and on the right hand side being equal.

Since Putinar's Positivestellensatz does not give a degree bound on the coefficients we have to look at a relaxation of the previous problem. Fix $d \in \mathbb{N}$

$$
\begin{aligned}
& \rho_{d}=\sup _{\rho, \sigma_{0}, \sigma_{1}, \ldots, \sigma_{m}} \rho \\
& \text { s.t. } p-\rho=\sigma_{0}+\sum_{i} \sigma_{i} g_{i} \\
& \quad \sigma_{0} \in \Sigma_{2 d}[\boldsymbol{x}], \sigma_{i} \in \Sigma_{2\left(d-d_{g_{i}}\right)}[\boldsymbol{x}] .
\end{aligned}
$$


Let $w_{k}$ be the vector of monomials up to degree $k$ in the variables $\boldsymbol{x}$ then (7) can be formulated as the following semidefinite program (SDP) ${ }^{7}$

$$
\begin{aligned}
& \sup _{\rho, M_{0}, M_{1}, \ldots, M_{m}} \rho \\
& \text { s.t. } p-\rho=w_{d}^{T} M_{0} w_{d}+\sum_{i} w_{d-d_{g_{i}}}^{T} M_{i} w_{d-d_{g_{i}}} g_{i} \\
& M_{0} \in \mathbb{R}^{\left(\begin{array}{c}
n+d \\
d
\end{array}\right)}, M_{i} \in \mathbb{R}^{\left(\begin{array}{c}
n+d-d g_{i} \\
d-g_{g_{i}}
\end{array}\right) \times\left(\begin{array}{c}
n+d-d g_{i} \\
d-d_{g_{i}}
\end{array}\right)} \\
& M_{0} \succcurlyeq 0, M_{i} \succcurlyeq 0 .
\end{aligned}
$$

Again note that the equality in (8) is an equality as functions. Thus we have only to compare coefficients. The equality constraints we obtain this way are linear. In particular $\boldsymbol{x}$ is not a variable in the above optimization problem.

The relaxation (8) gives in general a lower bound on the objective of (4). We have the following theorem relating the solutions of (8) to (6).

Theorem 2 (Lasserre 2010b, Th.5.6) Let the assumptions of Putinar's Positivstellensatz hold. Then the optimal solution of the relaxed problem $\rho_{d}$ converges for $d \rightarrow \infty$ to the optimal solution.

\section{Reformulating the GNEP}

We now return to the GNEP problem from Sect. 1. We have to deal with the parametrized optimization problem of each agent. In Lasserre (2010a) the dual problem is considered.

Fix $v$ and $d \in \mathbb{N}$ sufficiently large. We look at player $v$ 's optimization problem

$$
\begin{aligned}
& \min _{x^{v}} \theta_{v}\left(x^{v}, x^{-v}\right) \\
& \text { s.t. } h_{v}\left(x^{v}\right) \geq 0, g_{v}\left(x^{v}, x^{-v}\right) \geq 0
\end{aligned}
$$

We now regard $x^{-v}$ as a parameter and formulate a relaxation as in (7). We obtain the following parametrized optimization problem

$$
\begin{aligned}
& \sup _{\rho^{v}, M_{i}^{v}, N_{j}^{v}} \rho^{v} \\
& \text { s.t. } \theta_{\nu}\left(*, x^{-v}\right)-\rho^{v}=w_{d}^{T} M_{0}^{v} w_{d}+\sum_{i} w_{d-d_{g_{v}, i}}^{T} M_{i}^{v} w_{d-d_{g_{v}, i}} g_{v, i}\left(*, x^{-v}\right)+ \\
& \sum_{j} w_{d-d_{h_{v, j}}}^{T} N_{j}^{v} w_{d-d_{h_{v, j}}} h_{v, j}(*) \\
& M_{0}^{v} \in \mathbb{R}^{\left(\begin{array}{c}
n+d \\
d
\end{array}\right)}, M_{i}^{v} \in \mathbb{R}^{\left(\begin{array}{c}
n+d-d_{g_{v, i}} \\
d-d g_{v, i}
\end{array}\right) \times\left(\begin{array}{c}
n+d-d g_{v, i} \\
d-d g_{v, i}
\end{array}\right)}, M_{i}^{v} \succcurlyeq 0, \\
& N_{j}^{v} \in \mathbb{R}^{\left(\begin{array}{c}
n+d-d_{h_{v, i}} \\
d-d_{h_{\nu, i}}
\end{array}\right) \times\left(\begin{array}{c}
n+d-d_{h_{v}, i} \\
d-d_{h_{v, i}}
\end{array}\right)}, N_{i}^{v} \succcurlyeq 0 .
\end{aligned}
$$

\footnotetext{
7 See e.g. Boyd and Vandenberghe (2004).
} 
Note that, since $x^{-v}$ is regarded as a parameter, $d_{h_{v, j}}$ only refers to the degree of $h_{v, j}$ in the $x^{\nu}$. Furthermore in an abuse of notation let $w_{k}$ denote the monomials in the variables $x^{v}$ up to degree $k$. The $*$ signifies an equality as functions in the entries it replaces. Here this means that we compare the coefficients of the variables $x^{v}$ which themselves depend on the parameters $x^{-v}$. In particular those equations are polynomials in the variables $M_{i}^{v}, N_{j}^{v}, \rho^{v}$ and $x^{-v}$.

Now we use Proposition 1 and perform a change of coordinates replacing $M_{i}^{v}$ with $L_{i}^{v}\left(L_{i}^{v}\right)^{T}$ and $N_{j}^{v}$ with $T_{j}^{v}\left(T_{j}^{v}\right)^{T}$, where $L_{i}^{v}$ and $T_{j}^{v}$ are lower triangular matrices with nonnegative diagonal. This results in a system of polynomial equations and inequalities in the variables $\rho^{v}, L_{i}^{v}, T_{j}^{v}$ and $x^{-v}$.

For each $v$ we now have a problem of the form (10) but without the positive semidefinite constraint. To find an equilibrium we need an optimality condition or a relaxation thereof to replace the optimization. We propose the following:

Theorem 3 Let the assumptions of Putinar's Positivstellensatz hold and $\varepsilon>0, d \in \mathbb{N}$ sufficiently large. Furthermore let $\left(x^{-v}, \rho^{v}, L_{i}^{v}, T_{j}^{v}\right)$ for all $v, j, i$ be values in $\mathbb{R}$ satisfying the following polynomial system of equations and inequalities

$$
\begin{aligned}
& \theta_{v}\left(*, x^{-v}\right)-\rho_{d}^{v}=w_{d}^{T} L_{0}^{v}\left(L_{0}^{v}\right)^{T} w_{d}+ \\
& \sum_{i} w_{d-d_{g_{v, i}}^{T}}^{T} L_{i}^{v}\left(L_{i}^{v}\right)^{T} w_{d-d_{g_{v, i}}} g_{v, i}\left(*, x^{-v}\right)+ \\
& \sum_{j} w_{d-d_{h_{v, j}}}^{T} T_{j}^{v}\left(T_{j}^{v}\right)^{T} w_{d-d_{h_{v, j}}} h_{\nu, j}(*) \\
& \varepsilon \geq \theta_{v}\left(x^{v}, x^{-v}\right)-\rho^{v} \\
& g_{v, i}\left(x^{\nu}, x^{-v}\right) \geq 0 \\
& h_{v, j}\left(x^{v}\right) \geq 0 \\
& \left(T_{j}^{v}\right)_{l, l} \geq 0, \quad\left(L_{i}^{v}\right)_{k, k} \geq 0 \\
& L_{0}^{\nu} \in \mathbb{R}^{\left(\begin{array}{c}
n+d \\
d
\end{array}\right)}, L_{i}^{\nu} \in \mathbb{R}^{\left(\begin{array}{c}
n+d-d g_{v, i} \\
d-d g_{v, i}
\end{array}\right) \times\left(\begin{array}{c}
n+d-d g_{v, i} \\
d-d g_{v, i}
\end{array}\right)}, \\
& T_{j}^{v} \in \mathbb{R}^{\left(\begin{array}{c}
n+d-d_{h_{v}, i} \\
d-d_{h_{v}, i}
\end{array}\right) \times\left(\begin{array}{c}
n+d-d_{h_{v}, i} \\
d-d_{h_{v, i}}
\end{array}\right)}, \\
& L_{0}^{v}, L_{i}^{v}, T_{j}^{v} \text { lower triangular. }
\end{aligned}
$$

Then for all $v$ the point $x=\left(x^{1}, \ldots, x^{N}\right)$ satisfies the following inequality

$$
\left|\theta_{v}\left(x^{v}, x^{-v}\right)-\min _{y \in X_{v}\left(x^{-v}\right)} \theta_{v}\left(y, x^{-v}\right)\right| \leq \varepsilon
$$

Additionally let $\bar{x}$ be any equilibrium then there exists $k \in \mathbb{N}$ and $\rho^{v}, L_{i}^{v}, T_{j}^{v}$ for all $v$ such that $\bar{x}$ is a solutions to the above system of equations and inequalities. 
Proof For any feasible point $\hat{x}^{v}, \hat{\rho}_{d}^{v}, \hat{L}_{i}^{v}, \hat{T}_{j}^{v}$ we have that $\hat{\rho}_{d}^{v}$ is a lower bound on the optimum of $\theta_{v}\left(x^{v}, \hat{x}^{-v}\right)$. So

$$
\hat{\rho}_{d}^{v} \leq \min _{x^{v} \in X^{v}\left(\hat{x}^{-v}\right)} \theta_{v}\left(x^{v}, \hat{x}^{-v}\right) \leq \theta_{v}\left(\hat{x}^{v}, \hat{x}^{-v}\right)
$$

Since $\varepsilon \geq \theta_{\nu}\left(\hat{x}^{v}, \hat{x}^{-v}\right)-\hat{\rho}_{d}^{v}$, constraint (12), we obtain the following

$$
0 \leq \min _{x^{v} \in X^{v}\left(\hat{x}^{-v}\right)} \theta_{v}\left(x^{v}, \hat{x}^{-v}\right)-\hat{\rho}_{d}^{v} \leq \theta_{v}\left(\hat{x}^{v}, x^{-v}\right)-\hat{\rho}_{d}^{v} \leq \varepsilon
$$

Thus we know that $\theta_{v}\left(\hat{x}^{v}, \hat{x}^{-v}\right)$ satisfies the inequality (13).

If $\bar{x}$ is an equilibrium, then due to Theorem 2 we know that for any $\varepsilon>0$ and any $v$ there exists a $k_{v}$ such that $\theta_{v}\left(y, \bar{x}^{-v}\right)-\rho_{k_{v}}^{v}$ has a Putinar representation with $\left|\min _{y \in X_{v}\left(\bar{x}^{-v}\right)} \theta_{v}\left(y, \bar{x}^{-v}\right)-\rho_{k_{v}}^{v}\right|<\varepsilon$ and relaxation order $k_{v}$. Now we just have to set $k$ to the maximum of the $k_{v}$ and then $\bar{x}$ is feasible.

Once we computed a feasible point we can check whether it is a true equilibrium. To accomplish this we solve the polynomial optimization problem for each player to global optimality. We use Gloptipoly (Henrion et al. 2007) a program written for Matlab that employs the moment relaxation approach to solving polynomial optimization problems. This is the dual approach to the here presented sum of squares method.

Next we want to illustrate how to reformulate a GNEP into a system of equations and inequalities using our approach.

Example 1 We are looking at a simplified version of our model. To avoid confusion with exponents, we will write the players' number in the index. Let the number of players be two with objective function

$$
\begin{aligned}
\max _{a_{\nu}, q_{v}} & a_{\nu} q_{\nu}-k_{\nu} q_{\nu} \\
\text { s.t. } & g\left(a_{1}, q_{1}, a_{2}, q_{2}\right) \geq 0,
\end{aligned}
$$

where $\left(k_{1}, k_{2}\right)=\left(1, \frac{1}{2}\right)$ and $g\left(a_{1}, q_{1}, a_{2}, q_{2}\right)=1-a_{1}^{2}-q_{1}^{2}-a_{2}^{2}-q_{2}^{2}$. We obtain a relaxation of order 1 , i.e. the degree of $\sigma_{0}$ and $g_{i} \sigma_{i}$ does not exceed 2 . Therefore the multiplier of our constraint is just a nonnegative real number denoted my $m_{\nu}$. We look at the following equation.

$$
\begin{aligned}
& -a_{1} q_{1}+k_{1} q_{1}-\rho_{1}=w_{1}^{T} L L^{T} w_{1}+m_{1} g\left(a_{1}, q_{1}, a_{2}, q_{2}\right) \\
& -a_{2} q_{2}+k_{2} q_{2}-\rho_{2}=w_{2}^{T} M M^{T} w_{2}+m_{2} g\left(a_{1}, q_{1}, a_{2}, q_{2}\right),
\end{aligned}
$$

where $L=\left(\begin{array}{ccc}L_{1,1} & 0 & 0 \\ L_{2,1} & L_{2,2} & 0 \\ L_{3,1} & L_{3,2} & L_{3,3}\end{array}\right), \quad M=\left(\begin{array}{ccc}M_{1,1} & 0 & 0 \\ M_{2,1} & M_{2,2} & 0 \\ M_{3,1} & M_{3,2} & M_{3,3}\end{array}\right)$ and $w_{v}=\left(\begin{array}{c}1 \\ a_{v} \\ q_{v}\end{array}\right)$ 
We write out Eq. (15).

$$
\begin{aligned}
& -q_{1}+a_{1} q_{1}+\rho_{1}+L_{1,1}^{2}+2 a_{1} L_{1,1} L_{2,1}+a_{1}^{2} L_{2,1}^{2}+a_{1}^{2} L_{2,2}^{2} \\
& +2 q_{1} L_{1,1} L_{3,1}+2 a_{1} q_{1} L_{2,1} L_{3,1}+q_{1}^{2} L_{3,1}^{2}+2 a_{1} q_{1} L_{2,2} L_{3,2} \\
& +q_{1}^{2} L_{3,2}^{2}+q_{1}^{2} L_{3,3}^{2}+m_{1}-a_{2}^{2} m_{1}-a_{1}^{2} m_{1}-q_{2}^{2} m_{1}-q_{1}^{2} m_{1}=0
\end{aligned}
$$

Comparing coefficients in $a_{1}, q_{1}$ and adding the other constraints gives for player one the following equations and inequalities.

$$
\begin{aligned}
\rho_{1}+L_{1,1}^{2}+2 m_{1}-a_{2}^{2} m_{1}-q_{2}^{2} m_{1} & =0 \\
-1+2 L_{1,1} L_{3,1} & =0 \\
2 L_{1,1} L_{2,1} & =0 \\
L_{3,1}^{2}+L_{3,3}^{2}-m_{1} & =0 \\
L_{2,1}^{2}+L_{2,2}^{2}-m_{1} & =0 \\
1+2 L_{2,1} L_{3,1}+2 L_{2,2} L_{3,2} & =0 \\
m_{1} & \geq 0 \\
g\left(a_{1}, q_{1}, a_{2}, q_{2}\right) & \geq 0 \\
a_{1} q_{1}-q_{1}+\rho_{1} & =0
\end{aligned}
$$

For $\varepsilon=0$ and relaxation order 1 , we obtain the following equilibrium

$$
a_{1}=0.5, q_{1}=0.86602, a_{2}=0.00002, q_{2}=0.00323 .
$$

\section{Computational results}

We now return to the model of the New Zealand electricity spot market. First we present a real data set, second solve the model and lastly verify the solutions. All terms are with respect to a single time period which is a day.

The specific data for the HVDC Link in New Zealand are as follows: The power flow capacity of both arcs is $\bar{t}=16.8 \mathrm{GWh}$. Given the value of the electrical resistivity $/ \mathrm{km}$ at $20^{\circ} \mathrm{C}$ of $0.0139 \Omega / \mathrm{km}$, the length of transmission line of $607 \mathrm{~km}$ and the operating voltage of $350,000 \mathrm{~V}$ the result is $\lambda=0.0689$ for $t_{\{1,2\}}$ in GWh. Listed in Table 1 are all the coefficients for our electricity spot market model of New Zealand which are based on the Electricity Authority NZ (2011, 2010a). The demands and the capacities for the climate-based technologies corresponds to the average of the daily data of 2011.

We set $\varepsilon=0$ and $d=2$. We solve the model with the Ipopt solver in GAMS with a residual of $10^{-9}$. The running time is $17.45 \mathrm{~s}$ on an Intel E3-1290 with 8 GB of RAM. The results for bid, production and profit can be found in Table 2. We verified the results using GloptiPoly and SeDuMi.

With a homogeneous good one would usually expect a Bertrand competition. In contrast to this our simple model has a constant demand and so the consumer's demand is perfectly inelastic with respect to the price. Additionally we are capacity constrained 
Table 1 Coefficients for the New Zealand electricity spot market model based on real data

\begin{tabular}{llrlrlll}
\hline & Technology & $\kappa_{v}[\mathrm{GWh}]$ & $\eta_{v}[\mathrm{GJ} / \mathrm{GWh}]$ & $\phi_{v}[\$ / \mathrm{GJ}]$ & $\tau_{v}[\$ / \mathrm{GJ}]$ & $o_{v}[\$ / \mathrm{GWh}]$ & $\delta[\mathrm{GWh}]$ \\
\hline North & & 93.09 & & & & & 65.67 \\
& Coal & 16.84 & 10,500 & 4.006 & 0 & 9,600 & \\
& Diesel & 0.15 & 11,000 & 25.000 & 0 & 9,600 & \\
& Gas & 33.74 & $7,686.626$ & 6.500 & 1.0625 & 5,076 & \\
& Geothermal & 16.72 & 12,000 & 1.000 & 0 & 6,174 & \\
& Hydro & 20.18 & 3,600 & 1.000 & 0 & 0 & \\
& Wind & 4.52 & 3,600 & 1.000 & 0 & 12,038 & \\
& Wood waste & 0.93 & 12,000 & 2.000 & 0 & 11,800 & \\
South & & 47.02 & & & & & 38.69 \\
& Hydro & 46.54 & 3,600 & 1.000 & 0 & & \\
& Wind & 0.47 & 3,600 & 1.000 & 0 & 16,000 & \\
Total & & 140.12 & & & & & 104.46 \\
\hline
\end{tabular}

Table 2 Solution for the New Zealand electricity spot market model

\begin{tabular}{lllllll}
\hline \multirow{2}{*}{ North } & Technology & $\begin{array}{l}\text { Price offer } \\
{[\$ / G W h]}\end{array}$ & $\begin{array}{l}\text { Prod. offer } \\
{[\mathrm{GWh}]}\end{array}$ & $\begin{array}{l}\text { Cleared } \\
{[\mathrm{GWh}]}\end{array}$ & $\begin{array}{l}\text { Profit } \\
{[\mathrm{M} \$]}\end{array}$ & $\begin{array}{l}\text { Transport to } \\
{[\mathrm{GWh}]}\end{array}$ \\
& & & & & & 2.42 \\
& Coal & $63,206.1$ & 16.8 & 9.33 & 0.11 & \\
& Diesel & $28,233.1$ & 0 & 0 & 0 & \\
& Gas & $63,206.1$ & 28.99 & 14.64 & 0 & \\
& Geothermal & $35,127.3$ & 16.72 & 16.72 & 0.28 & \\
& Hydro & 34,159 & 20.18 & 20.18 & 0.62 & \\
& Wind & $63,206.1$ & 4.52 & 2.79 & 0.13 & \\
& Wood waste & $30,714.1$ & 0 & 0 & 0 & \\
South & & & & & \\
& & & & & & \\
& Hydro & $31,645.5$ & 46.54 & 41.51 & 1.16 & \\
& Wind & $32,260.5$ & 0 & 0 & 0 & \\
\hline
\end{tabular}

and so price equal marginal costs is out of question. Basically we are looking at a continuum of non obvious Cournot Equilibria where always some of the EPs do not offer their marginal costs.

The consumption-weighted average price of the here presented equilibrium is $40,712.3 \$ / G W h$ and is of the same magnitude as the reference node Stratford (SF D2201) with a consumption-weighted average price of 43,130.1 \$/GWh.

\section{References}

Aussel D, Correa R, Marechal M (2011a) Gap functions for quasivariational inequalities and generalized nash equilibrium problems. J Optim Theory Appl 151(3):474-488 
Aussel D, Correa R, Marechal P (2011b) Spot electricity market with transmission losses

Aussel D, Dutta J (2011) On gap functions for multivalued stampacchia variational inequalities. J Optim Theory Appl 149(3):513-527

Boyd S, Vandenberghe L (2004) Convex optimization. Cambridge University Press, Cambridge

Electricity Authority NZ (2010a) GEM: generation expansion model. Accessed: 12.10.2011

Electricity Authority NZ (2010b) Electricity industry participation code 2010 - Part 13. Version: 21.09.2012

Electricity Authority NZ (2011) CDS: centralised dataset. Accessed: 01.10.2012

Electricity Authority NZ (2012) Market operation service providers. http://www.ea.govt.nz/industry/ mo-service-providers/, Accessed: 01.10.2012

Facchinei F, Kanzow C (2007) Generalized nash equilibrium problems. 4OR Q J Oper Res 5(3):173-210

Henrion D, Lasserre J, Löfberg J (2007) GloptiPoly 3: moments, optimization and semidefinite programming. Optim Methods Softw 24(4-5):761-779

Hobbs B, Metzler C, Pang J-S (2000) Strategic gaming analysis for electric power systems: an mpec approach. IEEE Trans Power Syst 15(2):638-645

Jibetean D, de Klerk E (2006) Global optimization of rational functions: a semidefinite programming approach. Math. Program. 106:93-109

Lasserre JB (2010a) A "Joint+Marginal" approach to parametric polynomial optimization. SIAM J. Optim. 20:1995-2022

Lasserre JB (2010b) Moments, positive polynomials and their applications, vol 1 of Imperial College Press Optimization Series. Imperial College Press, London

Laurent M (2009) Sums of squares, moment matrices and optimization over polynomials, vol 149 of IMA volumes in Mathematics and its Applications. Springer, Berlin

Rosen JB (1965) Existence and uniqueness of equilibrium points for concave N-Person games. Econometrica 33(3):520-534

Tipler P, Gerlich D, Jerke G (2000) Physik, Volume 3. korr. Nachdr. of Spektrum Lehrbuch. Spektrum Akad. Verl 